\title{
Adsorption of Beryllium Atoms and Clusters on Graphene and in a Bilayer of G raphite Investigated by DFT
}

\section{Y ves Ferro $^{1}$, Nicolas Fernandez ${ }^{1}$, Alain Allouche ${ }^{1}$ and Christian L insmeier ${ }^{2}$}

${ }^{1}$ L aboratoire de Physique des Interactions Ioniques et M oléculaires A ix-M arseille U niversité /CNRS - UM R 7345, Campus de Saint Jérôme, Service 252, 13397 $M$ arseille Cedex 20, France

${ }^{2}$ M ax-Planck-Institut für Plasmaphysik, EURA TOM A ssociation, B oltzmannstrasse 2, 85748 Garching b. M ünchen, Germany

E-mail: yves.ferro@univ-amu.fr

\begin{abstract}
A bstract
We herein investigate the interaction of beryllium with a graphene sheet and in a bilayer of graphite by means of periodic DFT calculations. In all cases, we find the beryllium atoms to be more weakly bonded on graphene than in the bilayer. $\mathrm{Be}_{2}$ forms both magnetic and non-magnetic structures on graphene depending on the geometrical configuration of adsorption. We find the stability of the Be/bilayer system to increase with the size of the beryllium clusters inserted into the bilayer of graphite. We also find a charge transfer from beryllium to the graphite layers. All these results are analyzed in terms of electronic structure.
\end{abstract}

PACS : $68.43 . \mathrm{Fg}, 68.65 . \mathrm{Pq}, 73.20 . \mathrm{At}$ 


\section{Introduction}

The present paper focuses on the beryllium-graphite interaction, with the aim to provide detailed mechanisms, fundamental data and a comprehensive understanding of the chemical reactivity of beryllium atoms toward graphite. We therefore investigate the interaction of Be atoms and clusters with graphene and in a bilayer of graphite. Interaction energies are calculated for various physical situations - magnetic and non-magnetic structures, weak (physisorption) and strong (chemisorption) interactions. The results are discussed on the basis of electronic structure analysis and orbital theory. As in previous works [1-5], results are extrapolated from graphene to the surface of graphite, and from the bilayer to the bulk of graphite. This paper is the first part of a more global investigation dedicated to understand the formation of beryllium carbide from deposited beryllium atoms on graphite. It is conjointly led with experimental investigations, which results are discussed with the present ones later on in this paper.

Graphitic systems like pristine graphite, polyaromatic hydrocarbons $(\mathrm{PAH})$, graphene $[6,7]$, and carbon nanotubes [8] have attracted a renewed attention from a DFT point of view since the beginning of the twenty-first century. Their exceptional electronic properties, especially in the case of graphene and carbon nanotubes, are such that they are very promising materials for many technological applications, like in electronics for their semiconducting properties, fuel cells for their hydrogenabsorption capability, and in fusion science for their thermo-mechanical properties $[9,10]$. They are also expected to play a major role in the catalytic recombination of molecular hydrogen in the interstellar medium $[11,12]$. As a consequence, graphitic systems have been extensively studied by means of DFT [13].

Beryllium belongs to the group IIA of the alkaline earth metals. Its electronic configuration is $1 s^{2} 2 s^{2}$, which should not favour this element to form bonds with other atoms. However, the $2 s-2 p$ energy interval is small, 2 s electrons can partly be promoted to $2 p$ orbitals, which induces a significant degree of orbital mixing (hybridization) and favours the formation of covalent bonds. It has already been 
A dsorption of beryllium on graphene and graphite by DFT

demonstrated that, in the case of beryllium clusters, the degree of hybridization increases with the size of the cluster $[14,15]$. A nother consequence of the small $2 s-2 p$ energy interval is that the ground state of simple beryllium compounds is often multi-reference in nature, which is very challenging in computational chemistry. $\mathrm{M}$ any beryllium aggregates have also been investigated, like the $\mathrm{Be}_{3}$ trimer $[16,17]$ or bigger $B e_{n}$ clusters, with $n$ up to 9 , some of them at the M P4 [16] or FCI levels [17], some other with DFT [18-20].

The interaction of beryllium with graphitic molecular or periodic models has seldom been investigated. Based on molecular models, we review the works of Evangelisti et al. [21] who study the electronic and magnetic properties of $\mathrm{B}_{5}$ linear chain adsorbed on top of a large PAH by means of CASSCF and CASPT2 calculations, and that of Naumkin et al. [22] who investigated the interaction of a Be atom with ethylene at the M P2 level. Y et, ethylene is not graphite, we think of it as an interesting and minimal model for the interaction of beryllium with a simple $\pi$ system. Periodic models are almost exclusively investigated by DFT, since the use of post-HF calculations is prohibited in that case, with the exception of the CRY SCOR code [23, 24], which allows local M P2 calculations. Choi et al. [25] investigated the adsorption of Be atoms and other elements on the surface of periodic zigzag and armchair graphene nanoribbons (GNR). By means of DFT calculations and making use of the PBE functional [26], the authors found the interaction energies to be near zero at the centre of both GNRs where edge effects are expected to vanish. He et al. [27] studied the interaction of a beryllium dimer on the surface of graphene also using DFT-PBE. They found $\mathrm{Be}_{2}$ to chemisorb perpendicularly on the surface and to induce a magnetization of $1 \mu \mathrm{B} /$ cell.

One focus of the present paper is also to provide applied science on nuclear fusion with the fundamental data and mechanisms herein established about the beryllium-graphite system. Indeed, according to the current design, the materials facing the plasma in the nuclear fusion device ITER (International Tokamak Experimental Reactor) will be composed of graphite, beryllium and tungsten. Under operating conditions, these materials will be subject to heat and particle loads, beryllium will be 
sputtered or evaporated and will interact with the graphitic components. This interaction could lead to the modification of the electronic and mechanical properties of graphite, and as a consequence, there is great interest in better understanding the modification of the basic properties of graphite resulting from the beryllium interaction.

This paper is organized as follows. In section II, we present the method and computational details we used. In section III, the results we obtained are given in three different subsections devoted to the interaction of (i) a single $B$ e atom, (ii) $B e_{2}$, and (iii) small $B e_{n}$ clusters $(n=3,6,7)$ with graphite. These results are discussed in section IV with regards to previous theoretical works and existing experimental data on that system, before a conclusion is given in section $\mathrm{V}$.

\section{Computational details}

We here make use of DFT to investigate the interaction of beryllium atoms with one and two layers of graphene. The correct description of the van der Waals contributions responsible for the cohesion of the stacking of graphene layers is an important issue. Barone et al. [28] showed the PBE functional to accurately describe this interaction in graphite and between benzene molecules provided that the Grimme correction [29] for the van der W aals interactions is included (DFT-D2 scheme).

In the case of beryllium, the use of DFT is more debatable for small and weakly bonded systems where multi-reference states are expected to play an important role. These states require the post-HF multi-reference methods based on the interaction of configurations (IC) scheme to be accurately described. However, these methods are out of reach of complex systems, especially in the case of the solid-state theory. Fortunately DFT can succeed in giving a correct energetic description, since the exchange potential is local in DFT whereas it is non-local in Hartree-Fock (HF). Consequently, the DFT exchange contains the exact-exchange (HF exchange) plus a part of the non-dynamical (or leftright) correlation [30,31]. However, DFT is not accurate enough to predict the correct binding energy of the Be dimer, which is weak, about one hundred meV [32, 33]. It follows that a special attention is given in the following to $\mathrm{Be}_{2}$ adsorbed on the surface of graphene. However, the values of the energy we obtained are one order of magnitude higher than that of $\mathrm{Be}_{2}$, thus falling in the domain of DFT 
accuracy. Furthermore, it was found that DFT results adequately reproduce the experimentally observed trends for small $B e_{n}$ clusters despite the multi-reference nature of the wave function $[14,18$, 19], providing evidence of the soundness of DFT for the present work.

In a number of previous works, the interaction of Be with graphitic systems was investigated by DFT with the PBE functional $[25,27]$. In line with these authors, and including the van der Waals interaction in graphite according to the work of Barone et al. [28], all the calculations were performed with the Perdew-Burke-E rnzerhof generalized gradient approximation of the exchange and correlation functional, complemented by the Grimme correction DFT-D2 [29].

The Quantum Espresso code [34] developed in a plane-waves basis set was used with the Ultra-Soft pseudo-potentials [35] and energy cutoffs of $35 \mathrm{Ry}(476 \mathrm{eV})$ and $200 \mathrm{Ry}(2720 \mathrm{eV})$ for the truncation of the plane-wave expansion of the wave function and the electronic density, respectively. We used a $4 \times 4$ pseudo $2 \mathrm{D}$ supercell in which each image is repeated every $20 \AA$ along the $Z$ axis. The dimensions of the unit cell were optimized, cell parameters $\mathbf{a}=\mathbf{b}=2.463 \AA$ were obtained and subsequently used in all the calculations. The interlayer distance of the bilayer was set to its experimental value in graphite, i.e. $3.35 \AA$. All the atoms were allowed to relax during the geometry optimizations, except those at the border of the working-cell that simulate the constraints imposed by the infinite layer(s) on the cell. The Brillouin zone related to the $4 \times 4 \times 1$ supercell was sampled in a 6 $\times 6 \times 1$ mesh using the Monkhorst-Pack scheme [36], which is equivalent to a $24 \times 24 \times 1$ mesh in the unit cell. The Densities Of State (DOS) were plotted with a Gaussian broadening of 0.028 Ry, and population analyses were performed according to the Löwdin definition [37]. As the interpretation of the results relies on atomic and molecular orbitals interaction, the Khon-Sham orbitals were projected over the atomic wavefunctions of the pseudopotentials in order to produce projected Density Of States (pDOS).

Lastly and for the sake of simplicity, all interactions between beryllium and graphene (surface) or 
bilayers of graphite (bulk) are named adsorption energies in the rest of this paper they are defined as follows:

$$
\mathrm{E}_{\text {ads }}=\mathrm{E}_{\text {substrat }+n B e}-\mathrm{E}_{\text {substrat }}-n \mathrm{E}_{\mathrm{Be}}
$$

Binding energies were also used they are just minus the adsorption energy (- $\left.\mathrm{E}_{\text {ads }}\right)$.

\section{R esults}

\subsection{Single $B$ e atom}

\subsubsection{On graphene}

Figure la presents the potential energy curve of a single beryllium atom interacting with a graphene sheet. The most stable physisorption well is on top of a carbon ring at $3.120 \AA$ above the surface (figure 1b). The adsorption energy is weak, $E_{a d s}=-0.087 \mathrm{eV}$, which clearly indicates there is no chemical bonding. Other positions were tested for $\mathrm{Be}$, like on top of a carbon atoms where the adsorption energy and the equilibrium distance are similar to that of the previous case: $E_{\text {ads }}=-0.078$ $\mathrm{eV}, \mathrm{d}=3.262 \AA$. The projected DOS on the beryllium $2 \mathrm{~s}$ and $2 \mathrm{p}$ orbitals substantiates this finding (figure $1 c$ ). Only the $2 s$ orbital is occupied, and the empty $2 p$ energy levels remain above the Fermi level the electronic configuration of the beryllium ad-atom is $1 s^{2} 2 s^{2}$ as in the gas phase. A metastable configuration is also found below $1.8 \AA$, which probably arises from states crossing as already observed with the H-graphite system [38].

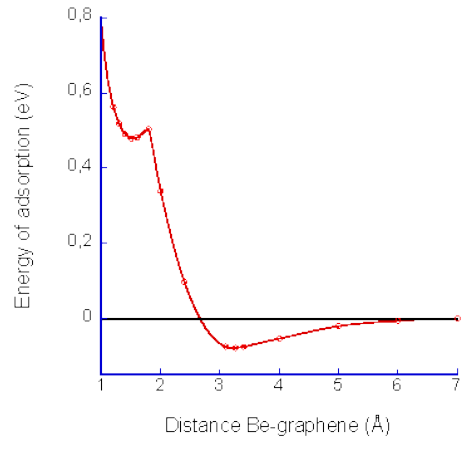

(a)

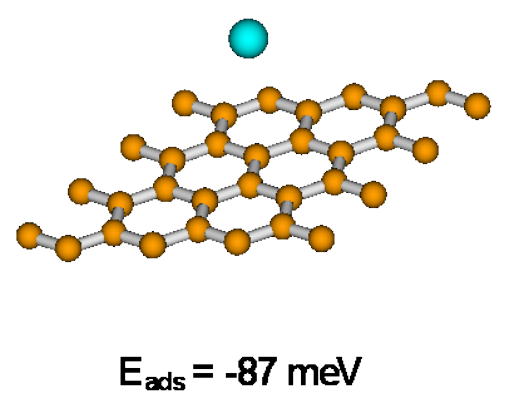

(b)

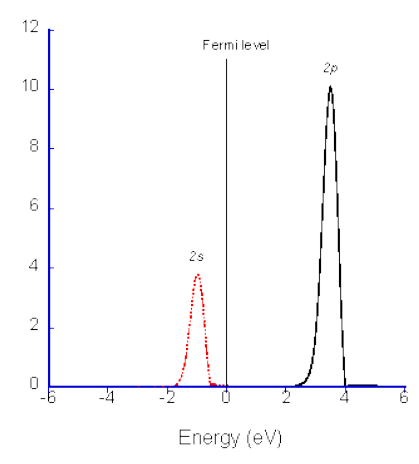

(c) 
Figure 1. Interaction of a Be atom with a graphene sheet. (a) Potential energy surface, (b) equilibrium geometry at adsorption, and (c) projected Density Of State ( $\mathrm{pDOS}$ ) on the $2 \mathrm{~s}$ and $2 \mathrm{p}$ orbitals of the $\mathrm{Be}$ atom.

\subsubsection{In the bilayer}

Beryllium is adsorbed inside a bilayer of graphite. Different starting geometry were used, with beryllium on top of both carbon atoms of each side of the bilayers, or on top of a C-C bond; all these calculations converged to the equilibrium geometry shown in figure $2 a$ where adsorption energy is $E_{\text {ads }}$ $=-1.027 \mathrm{eV}$ and the distance from the carbon atom on top of which Be resides is $1.789 \AA$. These results indicate a strong interaction between the bilayer and the beryllium atom; a chemical bond has been established. Figures $2 \mathrm{~b}$ and $2 \mathrm{c}$ show the DOSs of the pure graphite (bilayer) and of the bilayer plus Be. In the latter case, the Fermi level is shifted toward higher energies in the conduction band of the bilayer. This indicates a charge transfer from beryllium to the bilayer, which means doping of graphite. From figure $2 d$, all $2 s, 2 p_{x}, 2 p_{y}$ and $2 p_{z}$ orbitals are partly filled as can be seen from the projected DOS ( $\mathrm{pDOS}$ ): adsorption has induced Be orbitals mixing. The pDOS on the $\pi$ system of the

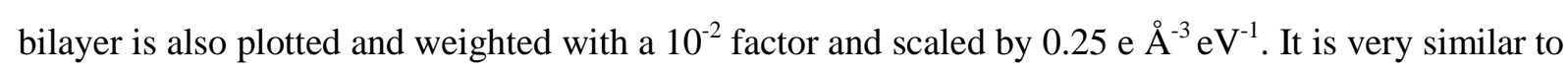
the pDOS on the pure bilayer (figure 2b), with the exception of the two peaks that appear just above $10 \mathrm{eV}$, and which also correspond to peaks in the projected DOS on $2 \mathrm{~s}$ and $2 \mathrm{p}_{\mathrm{z}}$ of beryllium; this is the signature of the interaction between the $\pi$-system of the bilayer and the $2 s$ and $2 p_{z}$ orbitals of beryllium.

Similarly, a peak is observed below $-20 \mathrm{eV}$ on the pDOS on $2 \mathrm{~s}$ and $2 \mathrm{p}_{\mathrm{z}}$ orbitals of $\mathrm{Be}$ (figure $2 \mathrm{~d}$ ) and on the total DOS of the bilayer plus beryllium (figure $2 \mathrm{c}$ ) this observation denotes an interaction between the $\sigma$-system of the bilayer and the $2 s$ and $2 p_{z}$ orbitals of beryllium. 


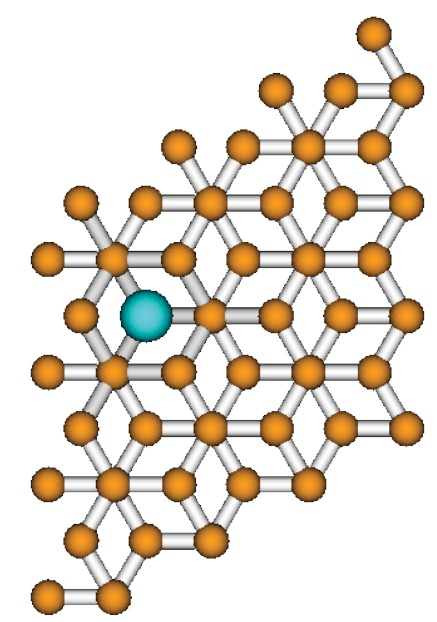

$E_{\text {ads }}=-1.027 \mathrm{eV}$

(a)

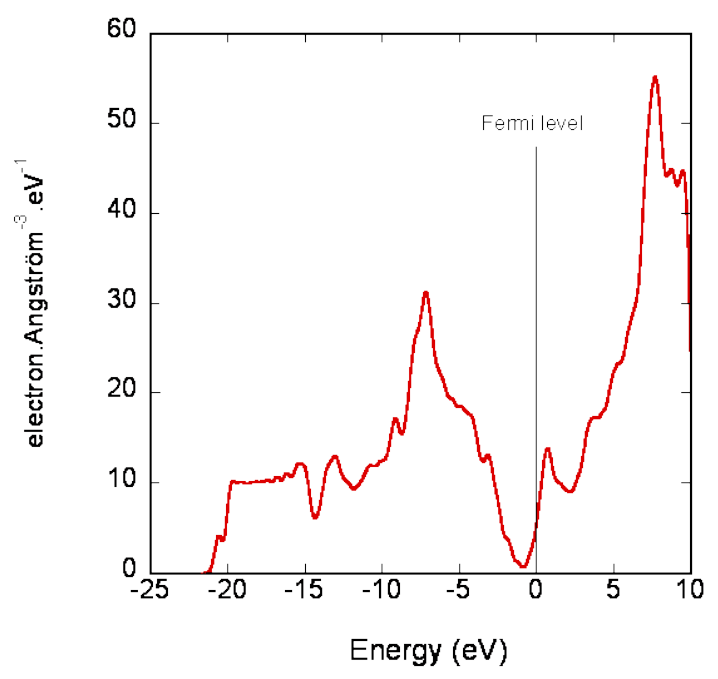

(c)

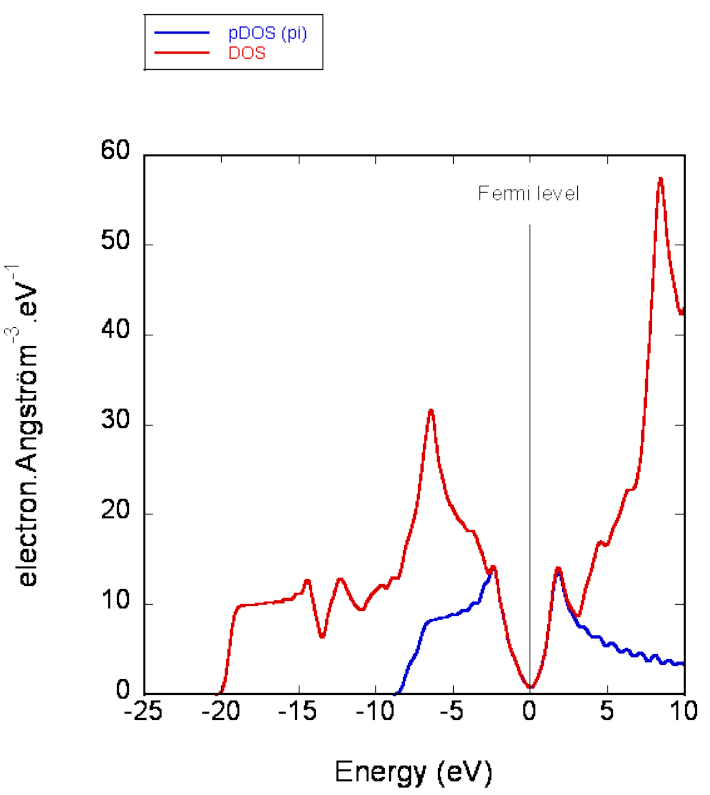

(b)

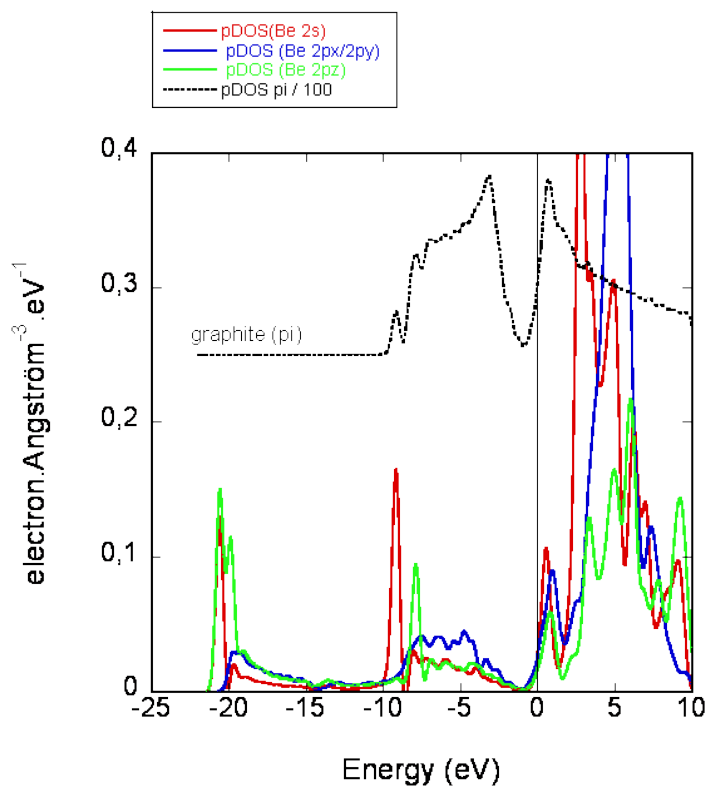

(d)

Figure 2. Interaction of a B e atom inside a bilayer of graphite. (a) Equilibrium geometry of adsorption, (b) DOS and projected DOS on the $\pi$ system of the bilayer of graphite without beryllium, (c) DOS of the bilayer plus a Be atom, (d) pDOS on the $2 s, 2 p_{x}, 2 p_{y}$ and $2 p_{z}$ orbitals of the Be atom and on the $\pi$ system of the bilayer plus a Be atom. The $2 p_{x}$ and $2 p_{y}$ orbitals are superimposed and cannot be distinguished. 


\subsection{The Be dimer}

\subsubsection{On graphene}

The binding energy of $\mathrm{Be}_{2}$ calculated within the PBE-D2 approach is $437 \mathrm{meV}$, far from the $116 \mathrm{meV}$ experimentally found [33]. However, the beryllium-graphite interaction energies here calculated are one order of magnitude higher than the $\mathrm{Be}_{2}$ binding energy. Some more, the PBE-D2 approach was checked on the $\mathrm{Be}_{2}$-benzene molecular model [39], which enables the more sophisticated MP2 calculation. The interaction energy calculated according to eq. 2 at the PBE-D2 and M P2 level with the aug-cc-pVTZ basis set are, in the singlet state, $-0.82 \mathrm{eV}$ and $-0.70 \mathrm{eV}$ respectively, and in the triplet state, $-0.93 \mathrm{eV}$ and $-0.82 \mathrm{eV}$, respectively. As a consequence, the interaction energy of $\mathrm{Be}_{2}$ with benzene is within 10 to $20 \%$ the accuracy of the M P2/aug-cc-pV TZ results. The inclusion of exact exchange to the PBE functional does not improve the results, since we found using PBE0 -1.11 eV and $-0.88 \mathrm{eV}$ for the triplet and singlet states, respectively. As a consequence, the PBE-D2 approach was found to be reliable to understand the basic mechanism at stake in the interaction of $\mathrm{Be}_{2}$ with graphene.

$$
E_{\text {int }}=E_{\text {Be2-benzene }}-E_{\text {benzene }}-E_{\text {Be2 }}[2]
$$

Hence, using PBE-D2, we find the beryllium dimer to bond onto graphene according to two stable configurations shown in Figure 3. Here again, different guesses were used as a starting point for the geometry optimization also different spin states were investigated. In the perpendicular configuration (Fig 3a), $\mathrm{Be}_{2}$ is adsorbed on top of a carbon ring along the $\mathrm{z}$ axis perpendicular to the surface. The $\mathrm{Be}-$ Be distance is $2.087 \AA$ and the adsorption energy is $E_{\text {int }}=-1.333 \mathrm{eV}(\mathrm{Eads}=-1.770)$. Charges are $1.9 \mathrm{e}$ and $1.8 \mathrm{e}$ for the upper and closer $\mathrm{Be}$ atom to the surface, respectively. The interatomic distance of $\mathrm{Be}_{2}$ in the gas phase is $2.43 \AA$ [33] the shorter interatomic distance we calculated indicates a strengthening of the dimer bonding. The distance from the surface to the closest Be atom is $d_{B \text { e-surface }}=1.404 \AA$, which is very short as compared to the monomer. This short $d_{\text {Be-surface }}$ along with the significant adsorption energy indicates chemical bonding to the surface. This state is magnetic with a magnetization of $1 \mu_{\mathrm{B}}$ /cell localized on the Be atom farthest from the surface, corresponding to a doublet spin state $S=1 / 2$, which is understood in the discussion section of the present paper. The triplet 
state $S=1$ was found to be $0.258 \mathrm{eV}$ higher in energy, with $E_{\text {int }}=-1.075 \mathrm{eV}$ and the same geometry, while the singlet state $S=0$ converged to the geometry of the parallel configuration.

In the parallel configuration (figure $3 \mathrm{~b}$ ), $\mathrm{Be}_{2}$ is adsorbed along the y axis parallel to the surface. The Be-Be distance is $1.966 \AA$ and the adsorption energy is $E_{i n t}=-0.811 \mathrm{eV}\left(E_{\text {ads }}=-1.027 e V\right)$. Charges are 1.8 e on both $B$ e atoms. The distance to $B e_{2}$ is $d_{\text {Be-surface }}=1.938 \AA$. These results al so indicate a strong Be-Be bond along with chemical bonding to the surface. In this case, the electronic configuration is non-magnetic consistent with a singlet spin state.

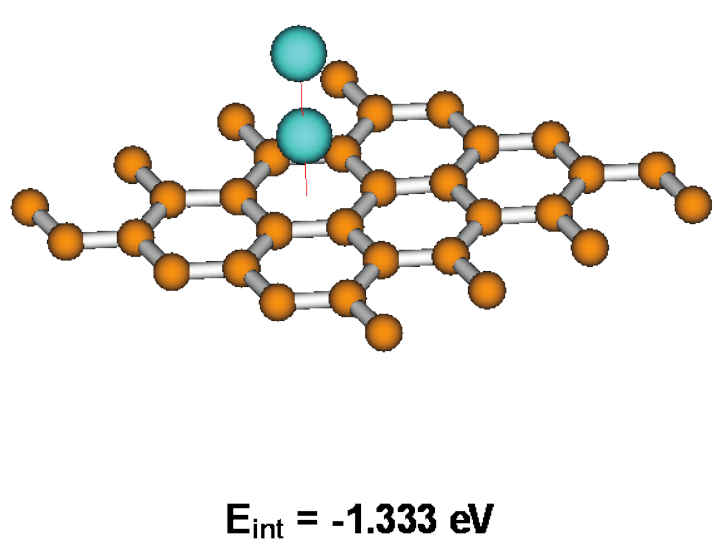

(a)

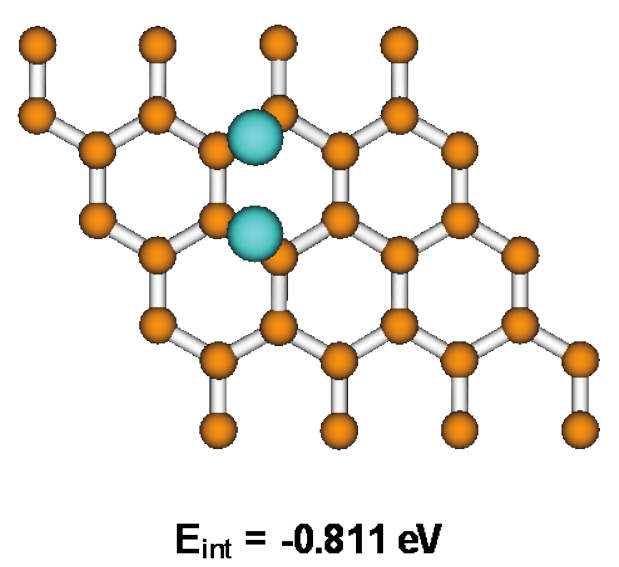

(b)

Figure 3. Equilibrium geometries of $\mathrm{Be}_{2}$ adsorbed on a graphene sheet (a) in a perpendicular configuration leading to magnetic state of $1 \mu \mathrm{B} / \mathrm{cell}$ and (b) in a parallel configuration that is a nonmagnetic state.

The electronic structure was then analysed in order to understand the origin of the two different kinds of bonding. To this end, figure 4 displays the band structures and pDOS of the magnetic dimer in the perpendicular configuration. Both are plotted in figure $4 a$ and $4 b$ for the spin-up component, and in figure $4 \mathrm{c}$ and $4 \mathrm{~d}$ for the spin-down component. $\mathrm{Be}_{2}$ belongs to the $\mathrm{D} \cdot{ }_{\mathrm{h}}$ point group, but the symmetry reduces to the $C_{6}$ point group in the geometry of adsorption. The orientation of the axis is as follows: $z$ 
is parallel to the $\mathrm{Be}_{2}$ molecular axis and perpendicular to the graphene layer, while $\mathrm{x}$ and $\mathrm{y}$ lies in the plane of graphene. The $\mathrm{C}_{6}$ representation reduces as $\Gamma_{2 \mathrm{~s}}=\mathrm{A} \oplus \mathrm{A}$ when built on the $2 \mathrm{~s}$ atomic orbitals of the beryllium dimer. Built on the $2 \mathrm{p}_{\mathrm{z}}$ atomic orbitals, it reduces according to $\Gamma_{2 \mathrm{pz}}=\mathrm{A} \oplus \mathrm{A}$, and on the set of $(2 \mathrm{px}, 2 \mathrm{py})$ orbitals, it reduces as $\Gamma_{(2 \mathrm{px}, 2 \mathrm{py})}=\mathrm{E}_{1} \oplus \mathrm{E}_{1}$. As a consequence, $\sigma$ molecular orbitals of $A$ symmetry mixing $2 s$ and $2 p_{z}$ beryllium orbitals are established as can be seen from figure $4 \mathrm{~b}$ and $4 d$ the associated bands are highlighted in red. Also $\pi$ molecular orbitals of $E_{1}$ symmetry mixing $2 p_{x}$ and $2 p_{y}$ Be orbitals are formed and are shown in yellow in figure 4 . Figure $4 a$ and $4 c$ show no dispersion associated with the bands involving beryllium, indicating local states centred on the Be dimer. Two $\sigma$ hybrids are located below the Fermi level for the spin-up component (figure 4a and 4c) only one $\sigma$ orbital is filled for spin-down, the other one is above the Fermi level (figure $4 \mathrm{c}$ and $4 \mathrm{~d}$ ). In all cases, $\pi$ orbitals from $2 p_{x}$ and $2 p_{y}$ are located above the Fermi level.

Figure 5 displays the band structures and pDOS of the non-magnetic dimer in the parallel configuration. In that case, the geometry of adsorption belongs to the $C_{2 v}$ point group. The orientation of the axis is as follows: $\mathrm{z}$ is perpendicular to the graphene layer, while $\mathrm{y}$ is parallel to the $\mathrm{Be}_{2}$ molecular axis. The $\mathrm{C}_{2 \mathrm{v}}$ representation reduces according to $\Gamma_{2 \mathrm{~s}}=\mathrm{A}_{1} \oplus \mathrm{A}_{2}$ when built on the $2 \mathrm{~s}$ atomic orbitals of the beryllium dimer. Built on $2 p_{y}$ and $2 p_{z}$, it reduces in much the same way $\left(\Gamma_{2 p y}=A_{1} \oplus A_{2}\right.$ and $\left.\Gamma_{2 \mathrm{pz}}=\mathrm{A}_{1} \oplus \mathrm{A}_{2}\right)$. Finally, built on $2 \mathrm{p}_{\mathrm{x}}$, it reduces according to $\Gamma_{2 \mathrm{px}}=\mathrm{A}_{2} \oplus \mathrm{B}_{1}$. A s a consequence, $\sigma$ hybrid molecular orbitals built on $2 s, 2 p_{y}, 2 p_{z}$ orbitals and belonging to the $A_{1}$ irreducible representation are established as can be seen in figure $5 b$, where they are highlighted in red. A part from that only to be found are other molecular orbitals of $A_{2}$ symmetry mixing $2 s, 2 p_{x}, 2 p_{y}$ and $2 p_{z}$ orbitals or of $B_{1}$ symmetry from $2 p_{x}$; some of them are shown in yellow in figure 5 . As a result, binding of the beryllium dimer on the surface of graphene in the parallel configuration is a consequence of the formation of a $\sigma$ hybrid molecular orbital of $A_{1}$ symmetry that points directly toward the $\pi$-system of the graphene surface. 


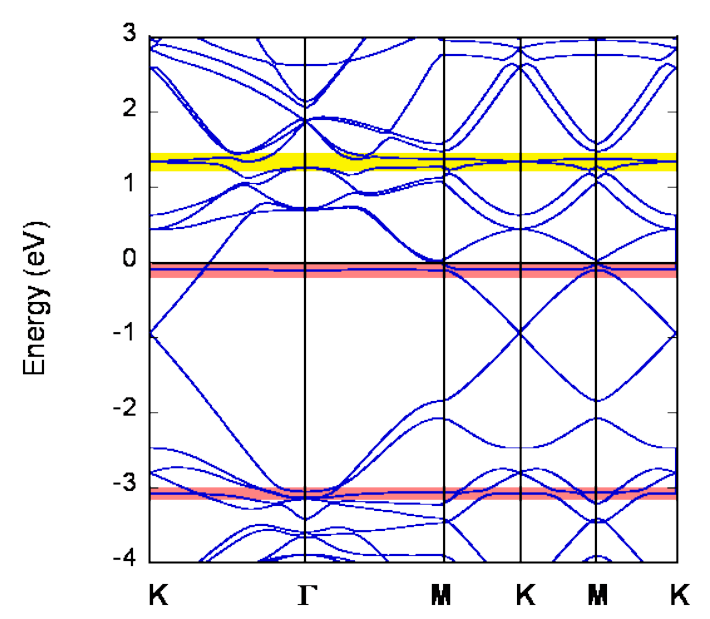

(a)

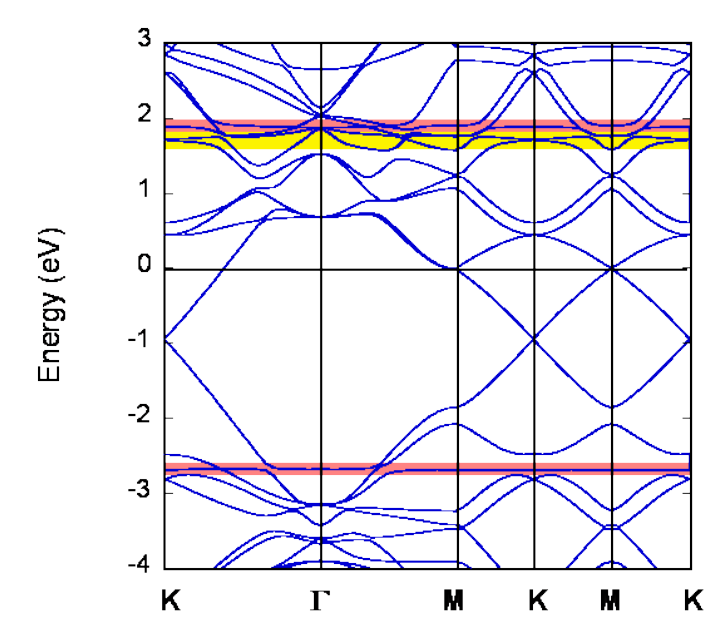

(c)

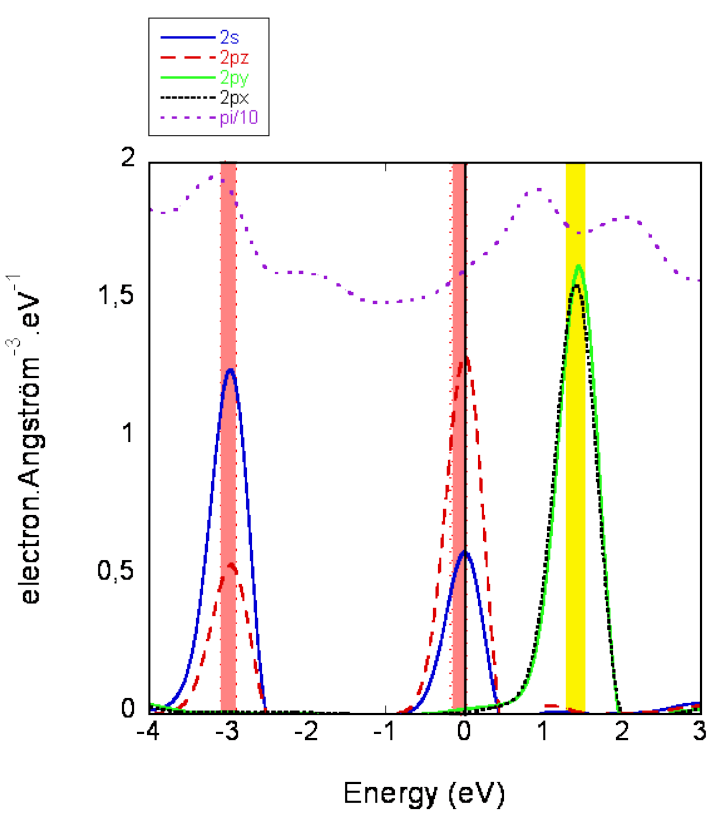

(b)

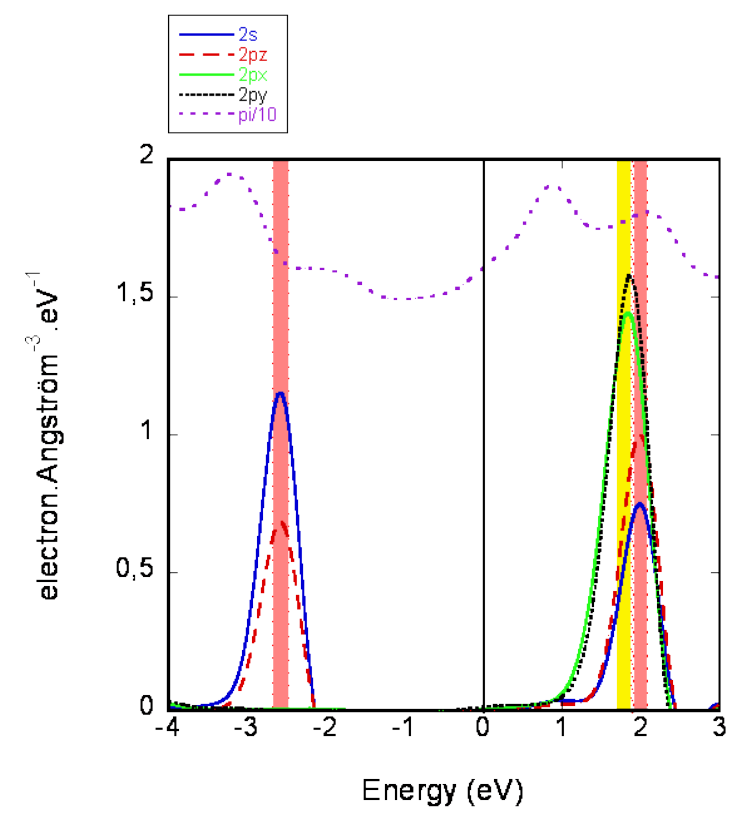

(d)

Figure 4: Electronic structure of $\mathrm{Be}_{2}$ adsorbed on a graphene sheet in the perpendicular configuration (magnetic state). (a) B and structure and (b) pDOS on $2 s, 2 p_{x}, 2 p_{y}$ and $2 p_{z}$ Be orbitals for the spin-up component. (c) Band structure and (d) pDOS on 2s, 2p $p_{x}$, $2 p_{y}$ and $2 p_{z}$ Be orbitals for the spin-down component. 


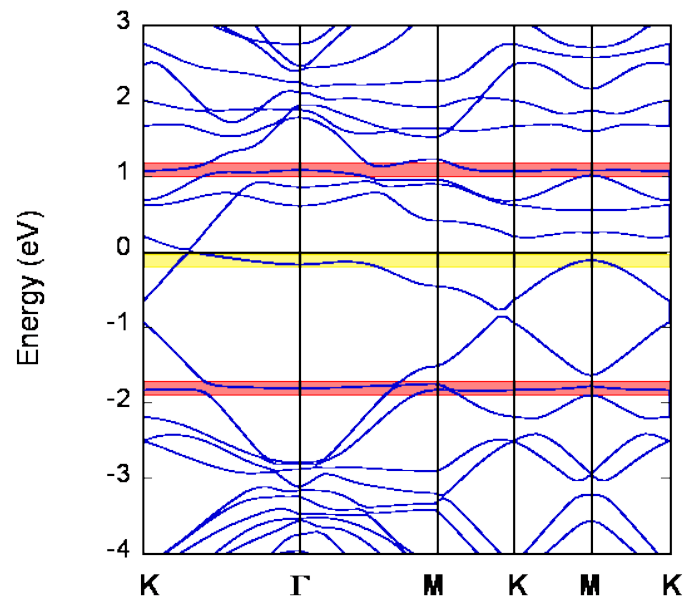

(a)

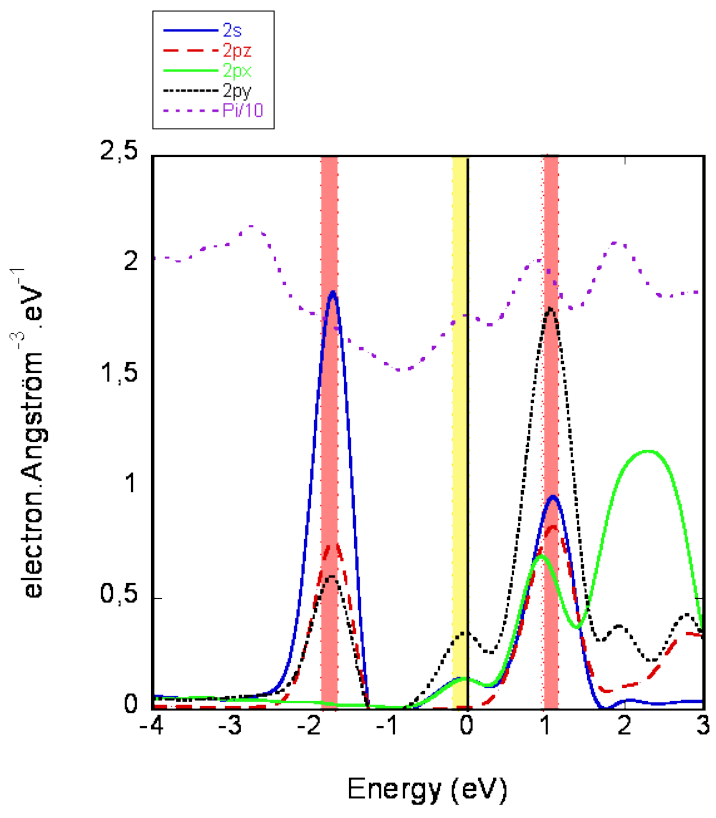

(b)

Figure 5. Electronic structure of $\mathrm{Be}_{2}$ adsorbed on a graphene sheet in the parallel configuration (nonmagnetic state). (a) B and structure and (b) pDOS on $2 s, 2 p_{x}, 2 p_{y}$ and $2 p_{z}$ B e orbitals.

\subsubsection{In the bilayer}

Figure 6 a displays the equilibrium geometry of a beryllium dimer adsorbed in a bilayer. The different starting geometry were both $\mathrm{B}$ e atoms on top of $\mathrm{C}$ of the same layer in ortho, meta and para positions. The equivalent of the parallel configuration on the surface (close to the meta position) is not found. The Be-Be distance is $2.232 \AA$, larger than on the surface, and close to that in the gas-phase. The adsorption energy is $E_{a d s}=-2.085 \mathrm{eV}(-1.042 \mathrm{eV} /$ atom), which is lower than that on the surface. Charges are 1.6 e on both Be atoms. Different spin configurations were calculated with spin multiplicity of $1,2,3(S=0,1 / 2,1)$ and relaxed (with incipient magnetization of half an electron on each Be atom); the ground state of the dimer was found to be singlet and non-magnetic. In figure $6 \mathrm{~b}$ and $6 \mathrm{c}$, we compare the pDOS of $\mathrm{Be}_{2}$ on graphene (parallel configuration) and in the bilayer in the domain of energy of the valence band. In both cases, the Fermi level is shifted in the conduction band of graphene as can be seen from the pDOS on the $\pi$ system. This shift indicates doping or charge transfer from beryllium to the graphene layer(s). This charge transfer is $0.74 \mathrm{e}$ to the bilayer and $0.47 \mathrm{e}$ to graphene according to the $L$ öwdin definition of charges. 


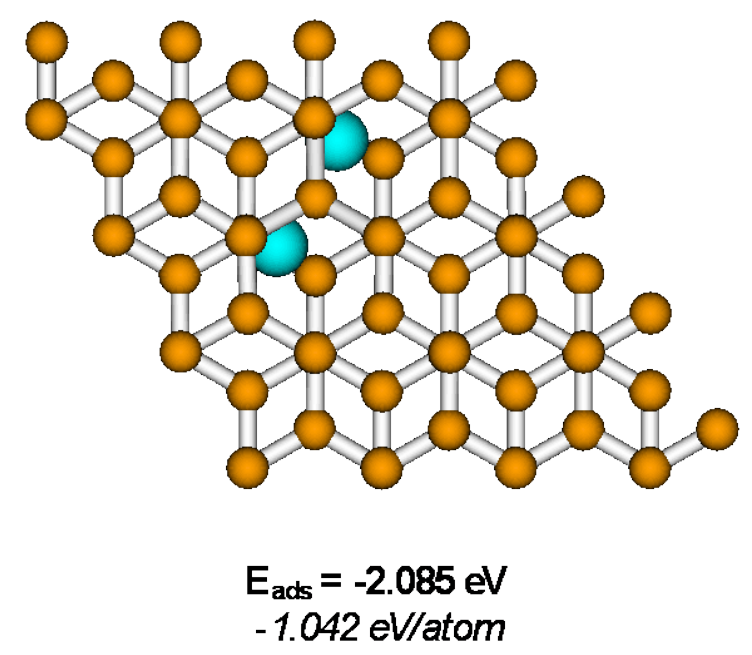

(a)

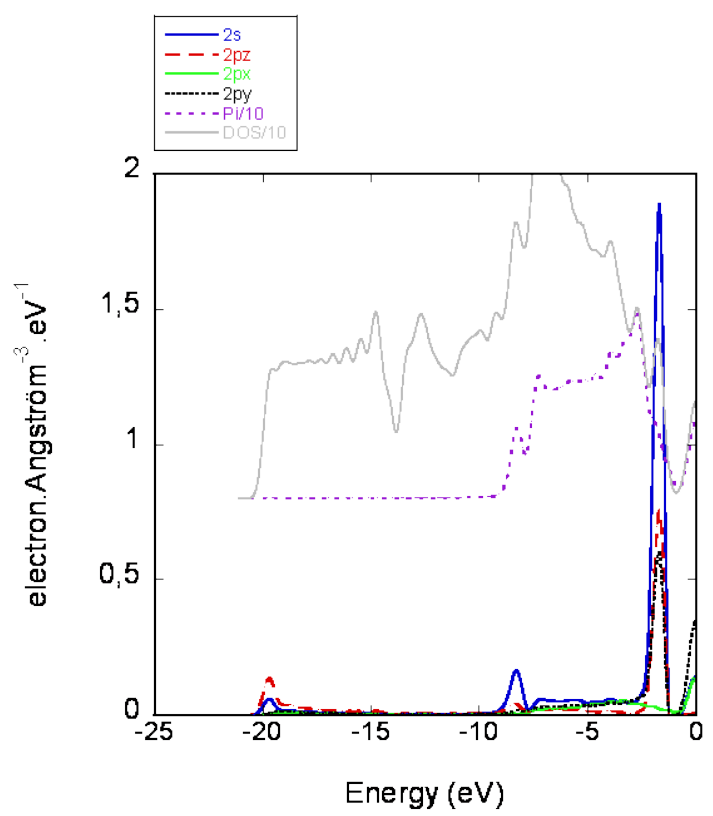

(b)

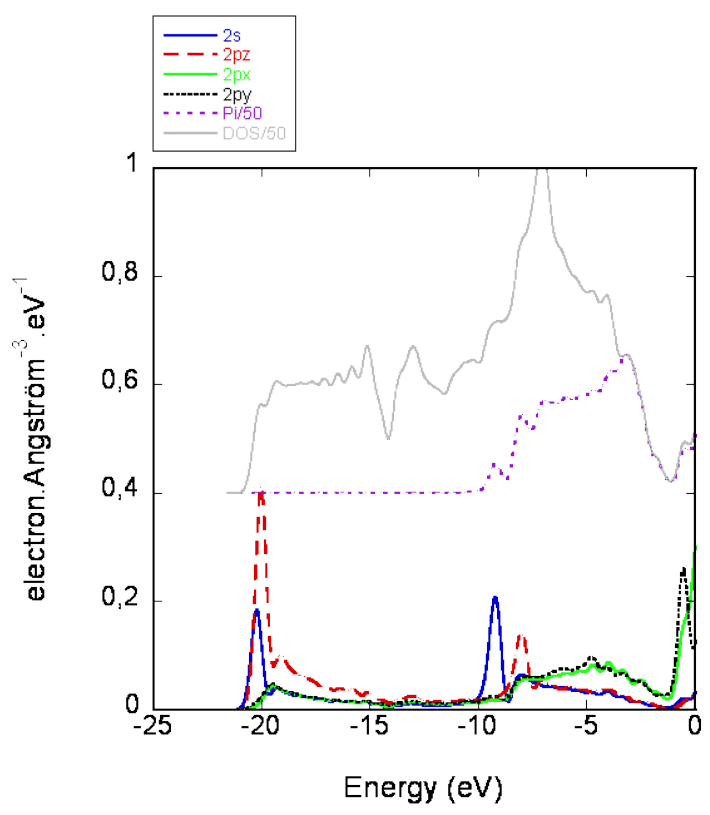

(c)

Figure 6. Beryllium dimer in a graphene bilayer. (a) Equilibrium geometry (b) pDOS of $\mathrm{Be}_{2}$ on a graphene sheet (parallel configuration), and (c) pDOS of $\mathrm{Be}_{2}$ in a bilayer. In both cases, DOSs are plotted in the domain of energy of the valence band. They are projected on the $2 s, 2 p_{x}, 2 p_{y}$ and $2 p_{z}$ orbitals of Be and on the $\pi$ system of graphene in (b) and of the bilayer in (c).

Let us now compare the adsorption of $\mathrm{Be}_{2}$ on the graphene surface in the parallel configuration and in the bilayer of graphite. The nature of the interaction is clearly different as can be seen from Fig $6 \mathrm{~b}$ and 6c. A $\sigma\left(2 s, 2 p_{y}\right.$ and $\left.2 p_{z}\right)$ peak around $-2.7 \mathrm{eV}$ is observed on the pDOS of the surface. This peak is 
A dsorption of beryllium on graphene and graphite by DFT

related to the $\sigma_{g}$ molecular orbital of $\mathrm{Be}_{2}$, which provides for the bonding of the dimer. In the bilayer, this peak vanishes while the contributions of the beryllium orbitals just above $-10 \mathrm{eV}$ and below - 20 $\mathrm{eV}$ become notably stronger than on the surface (figure $6 \mathrm{c}$ ). These contributions find their counterpart in the pDOS of the $\pi$-system and the total DOS, respectively. We therefore conclude that the Be-Be $\sigma$ bond tends to fade in the bilayer, and that beryllium is stabilized through the interaction of the $\pi$ system and $\sigma$-system with the $2 \mathrm{~s}$ and $2 \mathrm{p}$ orbitals.

\subsection{Beryllium clusters}

Figure 7 shows the equilibrium geometries of the $\mathrm{Be}_{3}, \mathrm{Be}_{6}$ and $\mathrm{B} \mathrm{e}_{7}$ clusters inserted inside the bilayer. We do no pretend here to be exhaustive with regard to all the stables structures of these clusters [19, 20]. Starting from different configuration with Be on top of carbon atoms or carbon rings, we privileged high symmetry configurations and high $\mathrm{Be}-\mathrm{Be}$ next nearest neighbours. The resulting clusters exhibits symmetries of order 3 for $\mathrm{Be}_{3}$ and $\mathrm{Be}_{6}$, and of order 6 for $\mathrm{Be}_{7}$. In all cases, the $\mathrm{Be}-\mathrm{Be}$ distance is short and close to $2.1 \AA$. Charges on $B$ e atoms are 1.7 e for $B e_{3}, 1.8$ e and 2.0 e at the corner and at the edge of $\mathrm{Be}_{6}$, and 1.8 e and 2.0 e for $\mathrm{Be}$ at the corner and the centre of $\mathrm{Be}_{7}$, respectively.

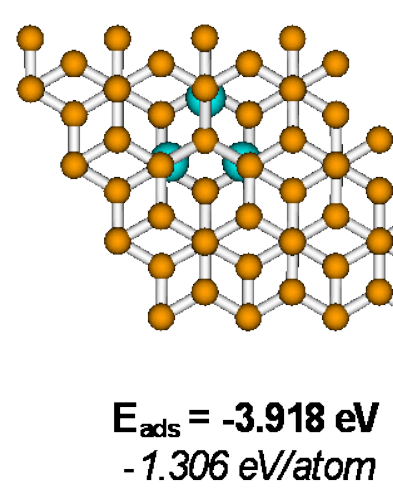

(a)

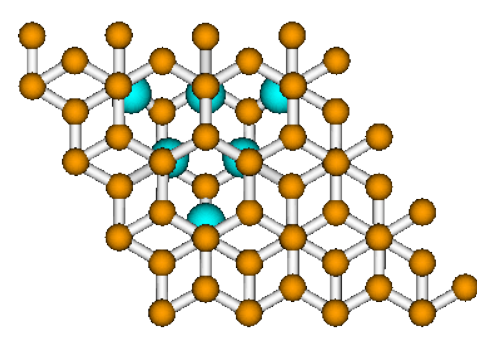

$$
E_{\text {ads }}=-9.048 \mathrm{eV}
$$$$
-1.508 \mathrm{eV} / \mathrm{atom}
$$

(b)

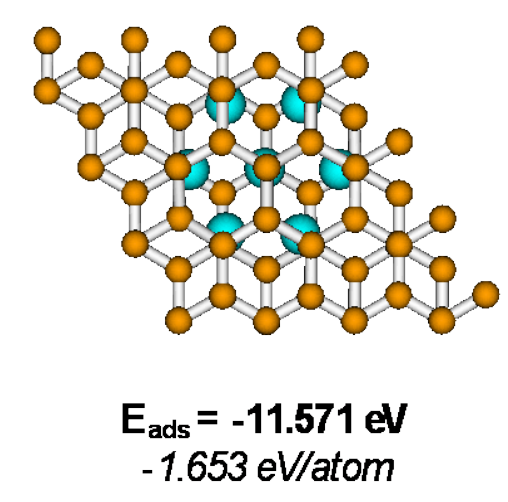

(c)

Figure 7. Equilibrium geometries of $\mathrm{Be}_{3}, \mathrm{Be}_{6}$ and $\mathrm{Be}$ clusters in a bilayer of graphite. 
In figure $8 \mathrm{a}$, the binding energy per $\mathrm{Be}$ atom is plotted versus the number $\mathrm{n}$ of atoms in the $\mathrm{B} \mathrm{e}_{\mathrm{n}}$ clusters points are oscillating around a linear trend shown in dashed line. We can expect the binding energy per Be atom to increase up to the cohesion energy of bulk beryllium, which we calculated to be $3.880 \mathrm{eV} /$ atom in an hexagonal compact packing. Figure 8a is a double y axis graph where is also plotted the mean number of nearest neighbours in the $B e_{n}$ series ( $y$ axis on the right of the graph). It is $0,1,2,3$ and 3.4 for $\mathrm{Be}, \mathrm{Be}_{2}, \mathrm{Be}_{3}, \mathrm{Be}_{6}$ and $\mathrm{Be}_{7}$, respectively. The trends of both curves are similar, indicating a possible correlation. All electronic structure involved here are spin singlet. Spin triplet were found to be $0.239 \mathrm{eV}, 0.226 \mathrm{eV}$ and $0.177 \mathrm{eV}$ higher in energy for $\mathrm{Be}_{3}, \mathrm{Be}_{6}$ and $\mathrm{B} \mathrm{e}_{7}$, respectively. Also relaxed spin electronic configuration were used with incipient magnetization of half an electron on each $\mathrm{B}$ e atom in order to insure the singlet spin state to be the true ground state.

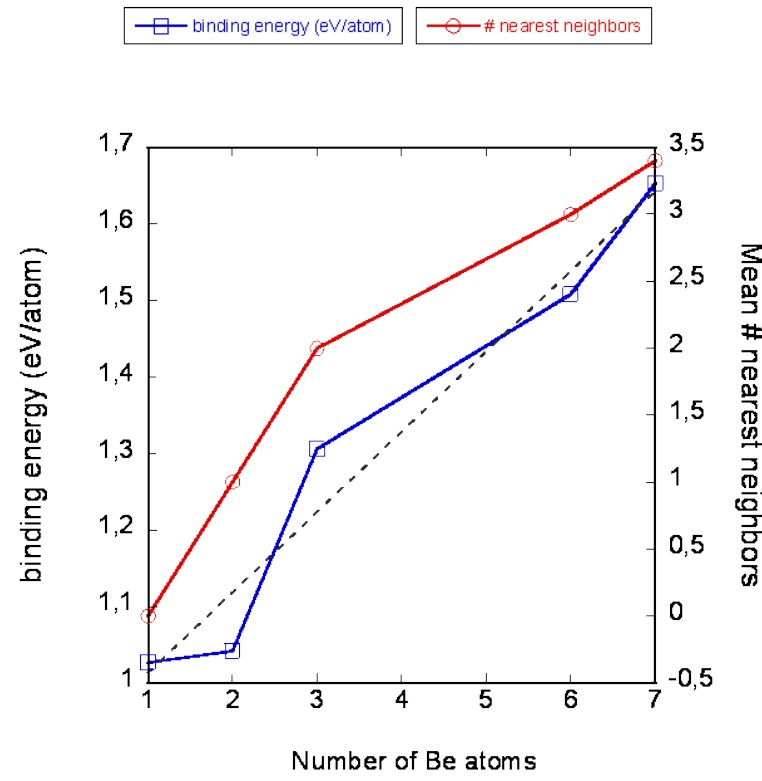

(a)

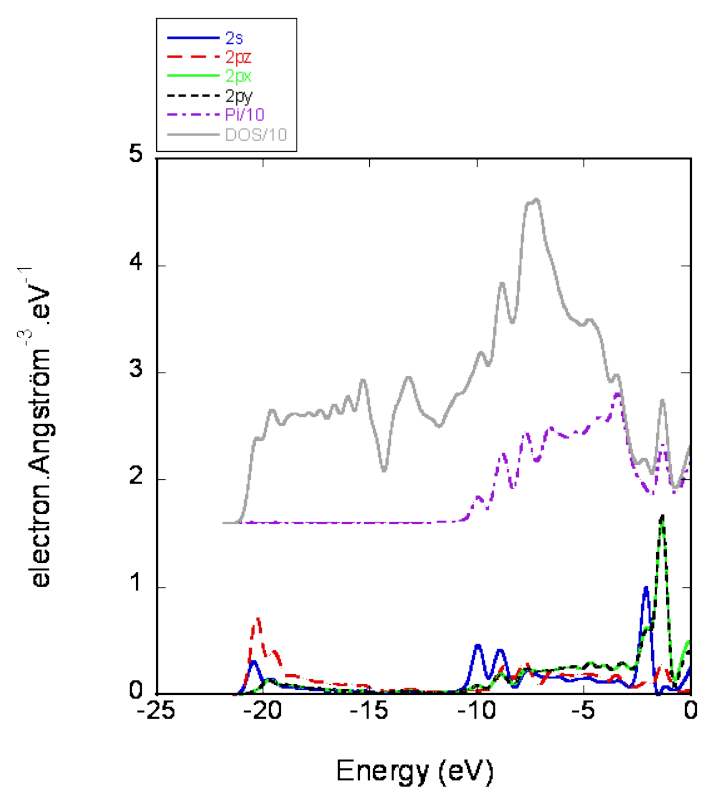

(b)

Figure 8. (a) D ouble y graph related to the adsorption of $B e_{n}$ clusters in a bilayer of graphite: binding energy (blue squares, left y axis) and the mean number of nearest neighbours (round circles, right $y$ axis) vs. the number of nearest neighbour in the cluster. (b) Total DOS and project DOS of $\mathrm{B} \mathrm{Be}_{7}$ cluster inside a bilayer of graphite.

The pDOS and total DOS of $\mathrm{Be}_{7}$ in the bilayer are plotted in figure 8b. The same behaviour as with $\mathrm{Be}_{2}$ (figure $6 \mathrm{c}$ ) is observed: $2 \mathrm{~s}$ and $2 \mathrm{p}$ orbitals of Be interact with the $\sigma$ and $\pi$ system below $-20 \mathrm{eV}$ 
A dsorption of beryllium on graphene and graphite by DFT

and above $-10 \mathrm{eV}$. Besides, $2 \mathrm{p}_{\mathrm{x}}$ and $2 \mathrm{p}_{\mathrm{y}}$ orbitals of beryllium interact with the $\pi$ system just below the Fermi level. A new feature appears in the case of $\mathrm{Be}_{7}$ as compared to $\mathrm{Be}_{2}$ : a $2 \mathrm{~s}$ peak around $-2.5 \mathrm{eV}$ is visible with no counterpart on the pDOS of the $\pi$ system. This peak means there is a Be-Be bond formation through $2 \mathrm{~s}$ orbitals, and is consistent with the increase in binding energy as the number of nearest neighbours rises as plotted in figure 8 a.

\section{Discussion}

As mentioned before in the introduction, the results we get are extrapolated from graphene to the surface, and from the bilayer to the bulk of graphite.

The interaction of a single beryllium atom with the surface of graphite leads to physisorption $\left(E_{a d s}=-\right.$ $0.087 \mathrm{eV}$ ). This result is in good agreement with previous ones concerning the adsorption of beryllium atoms on top of armchair-edged and zigzag-edged nanoribbons of graphite [25]. It s also in good agreement with calculations produced at the MP2 and $\operatorname{CCSD}(T)$ level on the Be-benzene system [39]. Contrary to the surface, the beryllium is chemically bonded to the bulk of graphite. The valence $2 \mathrm{~s}$ and $2 p$ orbitals of beryllium mix together and interact with the $\sigma$ and $\pi$ systems of graphite. As a result, binding energy in the bulk is about one order of magnitude stronger than on the surface.

The interaction of $\mathrm{Be}_{2}$ with the surface of graphite leads to two different configurations. When parallel to the surface, the non-magnetic electronic configuration of the beryllium dimer interacts with the nonmagnetic surface of graphite, thus resulting in a non-magnetic product whose electronic structure is described above. The case of the perpendicular configuration is more complex. In that case, He et al. [27] calculated the following binding energy, Be-Be and Be-surface distances: $1.04 \mathrm{eV}, 2.11 \AA$ and $1.49 \AA$, respectively. In this work, we find $1.333 \mathrm{eV}, 2.09 \AA$ and $1.40 \AA$, respectively. Otherwise, all calculation details are the same except for the Grimme correction. Hence, the higher binding energy and consequently shorter bond distances we find are the consequence of the van der Waals forces we include herein. He et al. [27] explained the strengthening of the Be-Be bond as compared to the free 
A dsorption of beryllium on graphene and graphite by DFT

dimer by the small $2 s-2 p$ interval in energy, which induces one electron to drop out of the $\sigma$ antibonding molecular orbital of the dimer. We totally agree with this interpretation, but wonder why the total magnetization yields $1 \mu_{B}$. Indeed, in another work, we investigated the adsorption of $\mathrm{H}$ dimers on the surface of graphite [40]. In that case, the dissociation of the electronic pair of the $\mathrm{H}-\mathrm{H}$ bond leads to $0 \mu_{B}$ or $2 \mu_{B}$ magnetization, depending on the adsorption site. This can be easily understood in terms of the angular momentum addition of spin $1 / 2$, leading to triplet and singlet spin states with $0 \mu_{\mathrm{B}}$ or $2 \mu_{\mathrm{B}}$ magnetizations, respectively. W hat is the mechanism leading to $1 \mu_{\mathrm{B}}$ magnetization in the case of the beryllium dimer? To answer this question, we analysed the electronic structure of $\mathrm{Be}_{2}$ on graphene using Hoffmann's methodology [41, 42]. Figure 9 shows an energy diagram of the Be $\mathrm{e}_{2}$-graphene interaction. On the left side of the diagram are given the molecular orbitals of the $\mathrm{Be}_{2}$ dimer with the $\sigma_{\mathrm{g}}$ bonding and $\sigma_{\mathrm{u}}$ anti-bonding molecular orbitals. These qualitative results are computed with the molecular GA USSIA N09 code [43] using the PBE DFT functional and the cC-PVTZ Dunning's basis set $[44,45]$. On the right side is given the DOS projected on the graphene $\pi$-system with the valence band shown in grey. The molecular levels of $\mathrm{Be}_{2}$ naturally interact with the whole $\pi$ bands of graphene of A symmetry. For the sake of simplicity, this whole interaction is modelled with the lowest energy level of the valence band, which belongs to the fully symmetric irreducible representation. This diagram is to be interpreted as follows:

- the $\pi$ energy level of the valence band of graphene is stabilized, which leads to the 1a energy level in the interacting scheme (middle of the diagram of figure 9),

- both $\sigma_{\mathrm{g}}$ and $\sigma_{\mathrm{u}}$ energy levels of $\mathrm{Be}_{2}$ are destabilized leading to the 2a and 3a energy levels in the interacting scheme. As a consequence, $3 a\left(\sigma_{u}\right)$ lies above the Fermi level, which induces one electron to jump from the $3 a\left(\sigma_{u}\right)$ level to the conduction band of graphene,

- the electronic relaxation that follows induces a stabilization of all the levels in energy including the single occupied $3 a\left(\sigma_{u}\right)$ level, which now lies below the Fermi level. 


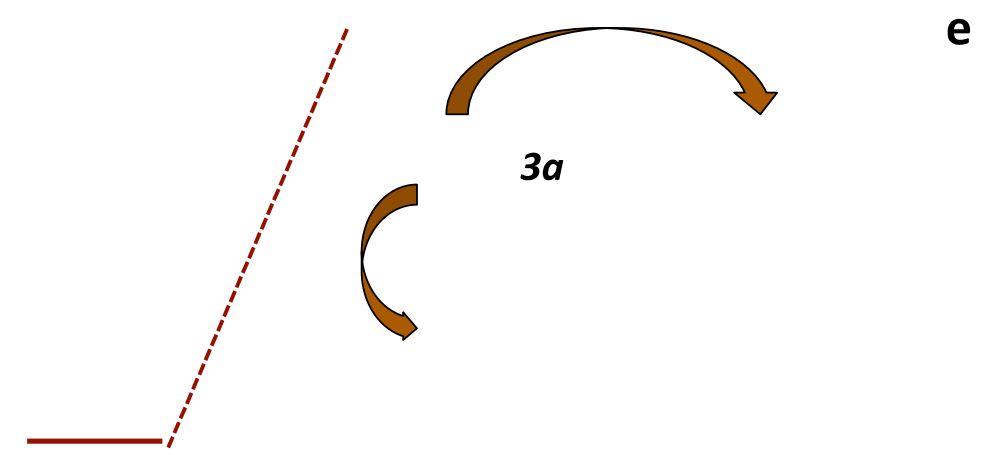

$2 a$

\section{$1 a$}

$\pi_{A 1}$

Figure 9. Energy diagram of the $\mathrm{Be}_{2}$-graphene interaction in the perpendicular configuration and magnetization of $1 \mu_{B}$. Blue square on the left side: labels of the $B e_{2}$ orbitals in the symmetry of the interacting system (bold) and of the free molecule (italic). On the right side: occupied (valence band in grey) and unoccupied (conduction band in white) pDOS of the $\pi$ system of graphene. The Be orbitals are interacting with the full levels of the $\pi$ system. For the sake of clarity, only shown is the interaction with an arbitrary low energy level of the $\pi$ system of graphene.

From the 4 electrons initially located on $\mathrm{Be}_{2}$, two initially in the bonding $\sigma_{\mathrm{g}}$ molecular orbital are in $2 \mathrm{a}$, one is in the antibonding $3 a$ (initially in $\sigma_{u}$ ), the last one is delocalized in the conduction band of graphene. This electron is thus delocalized on the whole $\pi$-system of the infinite surface. It has an associate spin density that tends toward zero, and its contribution to the magnetization of the adsorption site is zero. As a consequence, the only electron localized in the 3a orbital of the adsorption 
A dsorption of beryllium on graphene and graphite by DFT

site contributes to the magnetization with a magnitude of $1 \mu_{B}$, corresponding to a doublet electronic spin state.

We found in the literature [46] an example in which the use of a hybrid functional, HSE for instance $[47,48]$, switches the magnetic properties of a semi-metallic graphitic system to a non-magnetic insulator. This system is more precisely an heterogeneous edge decorated zigzag nano-ribbon of graphene with $\mathrm{OH}$ functional groups on one edge and $\mathrm{SO}_{2}$ groups on the other. When computed with the PBE functional, it is found to be spin-polarised and metallic. When computed with the hybrid HSE functional, it is no more spin-polarised and becomes a semi-conductor exhibiting band-gap. We checked in our case the spin-polarized properties we find have nothing to do with the use of hybrid or local DFT. To this end, the hybrid PBE 0 functional [49] was used to calculate the electronic properties of the Be2-graphene system in the same geometry as found for the perpendicular magnetic configuration with PBE. Both electronic configurations agree: a magnetization of $0.9 \mu_{B}$ is find with PBE 0, in agreement with the donation of one $\mathrm{Be}_{2}$ electron to the valence band of graphene. The bandgap of semi-conductors is known to be underestimated with local LDA and GGA functionals, while hybrids helps to restore its correct value. However, there is no band gap in our system, and the agreement between both PBE and PBEO seems reasonable.

In the bulk, $\mathrm{Be}_{2}$ interacts with graphite in a different manner. First, the 3D structure of graphite imposes geometry constraints that preclude the perpendicular configuration. Therefore, we compare the electronic structure of the parallel configuration on the surface with that of $B e_{2}$ in the bilayer. In Figure 6 , the $\sigma\left(2 \mathrm{~s}, 2 \mathrm{p}_{\mathrm{z}}\right)$ molecular orbital at $-2.7 \mathrm{eV}$ of $\mathrm{Be}_{2}$ on the surface (figure $6 \mathrm{~b}$ ) vanishes in the bilayer to the benefit of other contributions interacting with the $\sigma$ and $\pi$ structures of graphite (figure $6 \mathrm{c})$. We conclude to the bond breaking of $\mathrm{Be}_{2}$, and individual binding of each beryllium atom to the bilayer of graphite. The binding energy is about $1 \mathrm{eV}$ per beryllium atom, which is consistent with the binding energy of a single beryllium atom in the bilayer. 
The nature of the interaction of $\mathrm{Be}_{7}$ with the bilayer is the same as with $\mathrm{Be}_{2}$. Orbital mixing has already occurred and each beryllium atom is bonded to the graphite bilayer with an energy we estimate to be about $1 \mathrm{eV}$. The extra binding energy plotted in Fig $8 \mathrm{a}$ is the consequence of $\mathrm{Be}-\mathrm{Be}$ bonds that form through $2 \mathrm{~s}$ orbital mixing between $\mathrm{Be}$ atoms in the $\mathrm{Be}_{\mathrm{n}}$ clusters. This gain in energy with increasing $B e_{n}$ clusters size seems to confirm the capability of beryllium to form graphiteintercalated compounds (GIC). Indeed, GIC can be considered as infinity 2D clusters intercalated between 2 layers of graphite. Such a compound could serve as an efficient hydrogen storage material, which has already been proven by DFT in the specific case of $\mathrm{BeC}_{6}$ [50]. While the study of Ref 46 was conducted on an AB stacking of the graphene layers, other alkaline-earth metals like calcium were al ready shown to induce an A A stacking, which has been shown by $\mathrm{X}$-ray diffraction [51] on $\mathrm{CaC}_{6}$. We have checked the capability of the $\mathrm{Be}_{n}$ clusters to shift the bilayers from an $\mathrm{AB}$ stacking to an $\mathrm{AA}$ one. Considering the $\mathrm{n}=1,2,3,6,7$ series we found the following adsorption energies per $\mathrm{Be}$ atom for $\mathrm{AB}$ : $-1.027 \mathrm{eV},-1.042 \mathrm{eV},-1.306 \mathrm{eV},-1.508 \mathrm{eV},-1.653 \mathrm{eV}$, respectively. For $\mathrm{A} A$, we found: $-0.652 \mathrm{eV},-0.957 \mathrm{eV},-1.423 \mathrm{eV},-1.499 \mathrm{eV},-1.654 \mathrm{eV}$, respectively. Except from $\mathrm{Be}_{3}$, all of the clusters here considered are more or equally stable in an $A B$ than in $A A$ stacking. $A$ first explanation would be that $\mathrm{Be}_{\mathrm{n}}$ clusters do not form a uniform layer between two graphene as in $\mathrm{CaC}_{6}$. The beryllium concentration is smaller than the calcium concentration in $\mathrm{CaC}_{6}$, and the structure of graphite is consequently less distorted. A second one is about the electronic configuration of beryllium, which has no nd orbital available. Calcium 3 s orbitals donate electrons to the $\pi^{*}$ system of graphene, which is followed by a back donation from the $3 d$ orbitals of Ca to the $\pi^{*}$ system [52-54]. In the case of beryllium, we found no $2 \mathrm{~s}$ donation to the surface, while a charge transfer from $2 \mathrm{~s}$ to the $\pi^{*}$ system take place in the bilayer. However, no $\mathrm{d}$ orbital is available, which prevent the back donation to happen. As a consequence, the interaction is weaker $[50,52,54]$, and the distortion of the graphitic structure is smaller than in the case of calcium, thus favouring the natural AB stacking of graphite.

Experimentally, the interaction of Be atoms or clusters with carbon surfaces is very difficult to address due to the high reactivity of beryllium with oxygen species and the therefore very demanding 
requirements for a very good vacuum in the low $10^{-9} \mathrm{~Pa}$ range. Even at these low pressures, $\mathrm{Be}$ deposition is always accompanied by $\mathrm{BeO}$ deposition from the reaction of the $\mathrm{Be}$ vapour with the residual gas. Nevertheless, in experiments where Be is deposited from the vapour phase on graphite surfaces at room temperature starting with submonolayer coverage, the subsequent $\mathrm{X}$-ray photoelectron spectroscopy analysis of the Be 1s core level shows that a significant fraction of Be is in the carbidic state. For a nominal Be coverage of 0.1 monolayers, the Be 1s signal is composed of $30 \%$ $\mathrm{Be}_{2} \mathrm{C}, 20 \% \mathrm{BeO}$, and $50 \%$ metallic $\mathrm{Be}$. Since in these experiments it is not possible to determine whether the deposited beryllium at the surface is distributed atomically, or whether clusters of several atoms are present. Hence, it is difficult to assess the atomistic interaction, as discussed above from the DFT calculations. However, the detection of Be in the carbidic state (determined from the peak shift compared to the metallic Be 1s position) is a clear evidence for a chemical reaction between beryllium and carbon at room temperature [55]. The shift of the core level photoelectron peak demonstrates an electronic interaction between graphite and the adsorbed beryllium. Further details of the experimental investigations will be published in a separate paper.

\section{Conclusion}

We herein analysed the interaction of beryllium with graphite by means of DFT calculations the following results were established:

- Beryllium is weakly adsorbed on the surface of graphite, while it is chemically bonded into the bulk through orbital mixing and interactions with the $\sigma$ and $\pi$ systems of graphite.

- Adsorption of the beryllium dimer is favoured on the surface as a result of an electron transfer to graphite. In the bulk, the Be-Be bond dissociates and is replaced by a beryllium to graphite binding with an energy of about $1 \mathrm{eV}$ per Be atom.

- $\quad$ Large $B e_{n}$ clusters are stabilized the same way, in addition to which the effect of $B$ e-B e bonds that form between atoms of the cluster contribute extra binding energy to the system. 
A dsorption of beryllium on graphene and graphite by DFT

\section{Ackowledgements}

We wish to thank Pr Stephan Humbel and Dr Y annick Carrissan for the fruitful scientific discussions we had and which helped improving this paper. The authors of this work were granted access to the HPC resources of [CCRT/CINES/IDRIS] under the allocation i2012096612 made by GENCI (Grand Equipement National de Calcul Intensif). This work, supported by the European Communities under the partnering contract with EURATOM - CEA, and with the French Research Federation for fusion studies, was carried out within the framework of the European Fusion Development A greement. The views and opinions expressed herein do not necessarily reflect those of the European Commission.

\section{Bibliography}

[1] Ferro Y, M arinelli F and A llouche A 2002 J . Chem. Phys. 1168124

[2] Ferro Y, M arinelli F and A llouche A 2003 Chem. Phys. Lett. 368609

[3] Hornekær L, Sljivancanin Z, X u W , O tero R, Rauls E, Stensgaard I, Lægsgaard E, Hammer B and Besenbacher F 2006 P hys. Rev. Lett. 96156104

[4] Hornekær L, Rauls E, X u W, Sljivancanin Z, Otero R, Stensgaard I, L ægsgaard E, Hammer B and Besenbacher F 2006 P hys. Rev. Lett. 97186102

[5] Sljivancanin Z, A ndersen M , Hornekær L and Hammer B 2011 Phys. Rev. B 83205426

[6] Novoselov K S, Geim A K, M orozov S V, Jiang D, Zhang Y, Dubonos S V, Grigorieva I V and Firsov A. A 2004 Science 306666

[7] Meyer J C, Geim A K, Katsnelson M I, Novoselov K S, Booth T J and Roth S 2007 Nature (London) 46660

[8] Ijima M I 1991 Nature (London) 35456

[9] Philipps V , Roth J and Loarte A 2003 Plasma Phys. Control. Fusion 45, A 17

[10] Pégourié B et al 2009 J. Nucl. Mat. 390550

[11] Hollenbach D and Salpeter E E 1971 A strophys. J . 163155

[12] B urton M G, Bulmer M , M oorhouse A, Geballe T R and Brand P W J L 1992 M on. N ot. R. 
A dsorption of beryllium on graphene and graphite by DFT

\section{Astron. Soc. 2571}

[13] Ferro $Y$ and Allouche A 2009 Chemical reactivity and structural properties of the surface of graphite via Density Functional Calculations. "Q uantum Chemical Calculations of Surfaces and Interfaces of M aterials" page 315 (Editors: V . A . Basiuk and P. U gliengo). A merican Scientific Publishers

[14] Heaven M C, B ondybey V E, M erritt J M , K aledin A L 2011 C hem Phys Lett 5061

[15] Heaven M C, M erritt] M and B ondybey V E 2011 Ann. Rev. P hys. Chem 62375

[16] K aplan I G, Roszak S and L eszczynski J 2000 J. Chem. P hys 1136245

[17] J unquera-Hernandez J M, Sanchez-M arin J, Bendazzoli G L and Evangelisti S 2004 J. Chem. Phys 1208405

[18] B eyer M K, Kaledin L A, K aledin A L, Heaven M C and B ondybey V 2000 Chem. P hys. 26215

[19] Srinivas S and Jellinek J 2004 J. Chem. P hys 1217243

[20] Heaven M C, M errit] M, B ondybey V E, Annu. Rev. Phys. Chem 62375

[21] Evangelisti S, M onari A, L eninger T and Bendazzoli G L 2010 Chem. Phys. Lett. 496306

[22] Naumkin F Y 2010 Chem. P hys. Lett. 499203

[23] Pisani C, Busso M, Capecchi G, Casassa S, Dovesi R, M aschio L, Zicovich-W ilson C and Schütz M 2005 J. Chem. Phys. 122094133

[24] Pisani C, M aschio L, Casassa S, Halo M, Schütz M and U svyat D 2008 J . Comp. Chem. 292113 [25] Choi S M and J hi S H 2008 P hys. Rev. Lett. 101266105

[26] Perdew J P, B urke K and Ernzerhof M 1996 P hys. Rev. Lett. 773865

[27] He X, Chen Z X, Li Z and Zou Z 2010 J. Chem. Phys. 133231104

[28] Barone V , Casarin M , Forrer D, Pavone M, Sambi M and Vittadini A 2008 J . Comput. Chem. 30 934

[29] Grimme S 2006 ]. Comput. Chem. 271787

[30] M olawi K, Cohen A J and Handy N C 2002 Int. J. Quant. Chem. 8986

[31] Koch W and Holthausen M C, A Chemist's guide to Density F untional Theory 2002 Wiley-V CH, W einheim, p80

[32] B ondybey V E and English J H 1984 J. Chem. Phys 80568 
A dsorption of beryllium on graphene and graphite by DFT

[33] M erritt J M , Bondybey V E and Heaven M C 2009 Science 3241548

[34] Giannozzi P et al 2009 J . Phys.: Condens. M atter 21395502

[35] V anderbilt D 1990 P hys. Rev. B 417892

[36] M onkhorst H J and Pack J D 1972 Phys. Rev. B 135188

[37] Szabo A and Ostlund N S, Modern Quantum Chmistty 1989 McGraw-Hill

[38] Casolo S, Løvvik O M , M artinazzo R and Tantardini G F 2009 J. Chem. Phys. 130054704

[39] Fernandez N, Ferro $Y$, Carissan $Y$ and Allouche $A$, in preparation

[40] Ferro Y, Teillet-Billy D, Rougeau N, Sidis V, M orisset S and Allouche A 2008 Phys. Rev. B 78 085417

[41] Hoffmann R 1987 Angenw. Chem. Int. Ed. Engl. 26846

[42] Hoffmann R, Solids and Surfaces: A Chemist's View of Bonding in Extended Structures 1988 VCH, New Y ork

[43] Gaussian 09, Revision A.1, Frisch M J et al 2009, Gaussian, Inc., Wallingford CT

[44] Dunning T H J r 1989 J. Chem. Phys. 901007

[45] K endall R A, Dunning T H J r, Harrison R J 1992 J . Chem. Phys. 966769

[46] Wu M , Wu X, Cheng Zeng X 2010 J Phys. Chem. C 1143937

[47] Heyd J, Scuseria G E and Ernzerhof M 2003 J. Chem. Phys. 1188207

[48] Heyd J , Scuseria G E and Ernzerhof M 2006 J. Chem. Phys. 124219906

[49] A damo C and Barone V 1999 J. Chem. Phys. 1106158

[50] Cobin M and Iniguez J 2008 J. Phys.: Condens. M atter 20285212

[51] Emery N, Hérold C and Lagrange P 2005 J. Solid State Chem. 1782947

[52] Y oon M , Y ang S,Hicke C, W ang E, G eohegan D and Zhang Z 2008 P hys. Rev. Lett 100206806

[53] A taka C, A ktürk E and Ciraci S 2009 Phys. Rev. B 79 41406(R)

[54] Cazorla C, Shevlin S A and Guo Z X 2010 Phys. Rev. B 82155454

[55] Goldstrass P and Linsmeier Ch 2000 Nucl. Instrum. M eth. Phys. Res. B 161-163 411 
A dsorption of beryllium on graphene and graphite by DFT

\section{Captions}

Figure 1. Interaction of a Be atom with a graphene sheet. (a) Potential energy surface, (b) equilibrium geometry at adsorption, and (c) projected Density Of State ( $\mathrm{pDOS}$ ) on the $2 \mathrm{~s}$ and $2 \mathrm{p}$ orbitals of the Be atom.

Figure 2. Interaction of a B e atom inside a bilayer of graphite. (a) Equilibrium geometry of adsorption, (b) DOS and projected DOS on the $\pi$ system of the bilayer of graphite without beryllium, (c) DOS of the bilayer plus a Be atom, (d) pDOS on the $2 s, 2 p_{x}, 2 p_{y}$ and $2 p_{z}$ orbitals of the Be atom and on the $\pi$ system of the bilayer plus a Be atom. The $2 p_{x}$ and $2 p_{y}$ orbitals are superimposed and cannot be distinguished.

Figure 3. Equilibrium geometries of $\mathrm{Be}_{2}$ adsorbed on a graphene sheet (a) in a perpendicular configuration leading to magnetic state of $1 \mu \mathrm{B} /$ cell and (b) in a parallel configuration that is a nonmagnetic state.

Figure 4: Electronic structure of $\mathrm{Be}_{2}$ adsorbed on a graphene sheet in the perpendicular configuration (magnetic state). (a) B and structure and (b) pDOS on $2 \mathrm{~s}, 2 \mathrm{p}_{\mathrm{x}}, 2 \mathrm{p}_{\mathrm{y}}$ and $2 \mathrm{p}_{\mathrm{z}}$ Be orbitals for the spin-up component. (c) B and structure and (d) pDOS on $2 s, 2 p_{x}, 2 p_{y}$ and $2 p_{z} B$ e orbitals for the spin-down component.

Figure 5. Electronic structure of $\mathrm{Be}_{2}$ adsorbed on a graphene sheet in the parallel configuration (nonmagnetic state). (a) B and structure and (b) pDOS on $2 s, 2 p_{x}, 2 p_{y}$ and $2 p_{z}$ B e orbitals.

Figure 6. Beryllium dimer in a graphene bilayer. (a) Equilibrium geometry (b) pDOS of $\mathrm{Be}_{2}$ on a graphene sheet (parallel configuration), and (c) pDOS of $\mathrm{Be}_{2}$ in a bilayer. In both cases, DOSs are 
A dsorption of beryllium on graphene and graphite by DFT

plotted in the domain of energy of the valence band. They are projected on the $2 s, 2 p_{x}, 2 p_{y}$ and $2 p_{z}$ orbitals of Be and on the $\pi$ system of graphene in (b) and of the bilayer in (c).

Figure 7. Equilibrium geometries of $\mathrm{Be}_{3}, \mathrm{Be}_{6}$ and $\mathrm{B} \mathrm{e}_{7}$ clusters in a bilayer of graphite.

Figure 8. (a) D ouble y graph related to the adsorption of $B e_{n}$ clusters in a bilayer of graphite: binding energy (blue squares, left y axis) and the mean number of nearest neighbours (round circles, right y axis) vs. the number of nearest neighbour in the cluster. (b) Total DOS and project DOS of a Be cluster inside a bilayer of graphite.

Figure 9. Energy diagram of the $\mathrm{Be}_{2}$-graphene interaction in the perpendicular configuration and magnetization of $1 \mu_{B}$. Blue square on the left side: labels of the $B e_{2}$ orbitals in the symmetry of the interacting system (bold) and of the free molecule (italic). On the right side: occupied (valence band in grey) and unoccupied (conduction band in white) pDOS of the $\pi$ system of graphene. The Be orbitals are interacting with the full levels of the $\pi$ system. For the sake of clarity, only shown is the interaction with an arbitrary low energy level of the $\pi$ system of graphene. 